ARTICLE

Received 12 Sep 2016 | Accepted 28 Dec 2016 | Published 12 Apr $2017 \quad$ DOl: 10.1038/ncomms14430 OPEN

\title{
Multiscale structural and electronic control of molybdenum disulfide foam for highly efficient hydrogen production
}

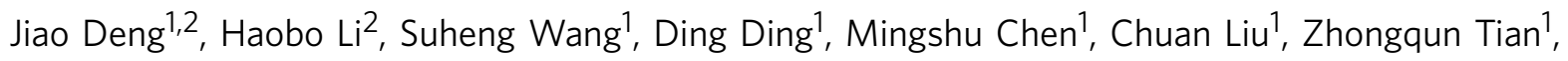
K.S. Novoselov ${ }^{3}$, Chao $\mathrm{Ma}^{4}$, Dehui Deng ${ }^{1,2} \&$ Xinhe $\mathrm{Bao}^{2}$

Hydrogen production through water splitting has been considered as a green, pure and highefficient technique. As an important half-reaction involved, hydrogen evolution reaction is a complex electrochemical process involving liquid-solid-gas three-phase interface behaviour. Therefore, new concepts and strategies of material design are needed to smooth each pivotal step. Here we report a multiscale structural and electronic control of molybdenum disulfide foam to synergistically promote the hydrogen evolution process. The optimized threedimensional molybdenum disulfide foam with uniform mesopores, vertically aligned twodimensional layers and cobalt atoms doping demonstrated a high hydrogen evolution activity and stability. In addition, density functional theory calculations indicate that molybdenum disulfide with moderate cobalt doping content possesses the optimal activity. This study demonstrates the validity of multiscale control in molybdenum disulfide via overall consideration of the mass transport, and the accessibility, quantity and capability of active sites towards electrocatalytic hydrogen evolution, which may also be extended to other energy-related processes.

\footnotetext{
${ }^{1}$ State Key Laboratory of Physical Chemistry of Solid Surfaces, Collaborative Innovation Center of Chemistry for Energy Materials (iChEM), College of Chemistry and Chemical Engineering, Xiamen University, Xiamen 361005, China. ${ }^{2}$ State Key Laboratory of Catalysis, Collaborative Innovation Center of Chemistry for Energy Materials (iChEM), Dalian Institute of Chemical Physics, Chinese Academy of Science, Dalian 116023, China. ${ }^{3}$ School of Physics and Astronomy, University of Manchester, Oxford Road, M13 9PL Manchester, UK. ${ }^{4}$ Center for High Resolution Electron Microscopy, College of Materials Science and Engineering, Hunan University, Changsha 410082, China. Correspondence and requests for materials should be addressed to D.D. (email: dhdeng@dicp.ac.cn) or to X.B. (email: xhbao@dicp.ac.cn).
} 
T he properties of two-dimensional (2D) $\mathrm{MoS}_{2}$ are significantly different in comparison with its three-dimensional (3D) form. Thus, it has been considered for a number of applications, such as solar cells ${ }^{1-3}$, photocatalysis ${ }^{4-6}$, lithium ion batteries $^{7-9}$ and electrocatalysis ${ }^{10-13}$. Owing to its natural abundance, low cost and good catalytic performance, recently $\mathrm{MoS}_{2}$ has become a representative non-precious material for electrocatalytic hydrogen evolution reaction (HER) of water splitting ${ }^{14-21}$. Such liquid-to-gas electrochemical conversion, with a complex reaction process at the interface of liquid $\left(\mathrm{H}^{+}\right)$, solid (catalyst) and gas $\left(\mathrm{H}_{2}\right)$, require a multiscale structural and electronic control of $\mathrm{MoS}_{2}$ to make each involved reaction step to proceed smoothly. This includes sufficient transport of reactants and products, accessibility of catalyst surface, abundant active sites and enough catalytic capability. Similar to the recent developments in the mesoporous framework of graphene ${ }^{22-24}$ or polymer $^{25,26}$ foam, the design and preparation of a uniform mesoporous $\mathrm{MoS}_{2}$ foam could simultaneously facilitate the mass transport and accessibility of active sites. Yet, unlike the flexibility of carbon atoms skeleton in graphene and organic small molecules in polymers, $\mathrm{MoS}_{2}$ with single-crystal layer composed of three molecular layers (S-Mo-S) appears much more inflexible, which leads to such engineering still remaining a great challenge. Also, the S-edges of $2 \mathrm{D} \mathrm{MoS}_{2}$ is usually considered as the active sites, while the in-plane structure is not active in catalysis ${ }^{15,27-31}$. Our recent work demonstrated that introducing different dopant atoms into the $\mathrm{MoS}_{2}$ matrix can enhance the intrinsic activity of its in-plane $\mathrm{S}$ atoms ${ }^{32}$. Therefore, further atomic-scale engineering via doping hetero atoms into the mesoporous $\mathrm{MoS}_{2}$ foam may achieve a multiscale modulation to synergistically boost the HER electrochemical process. However, such all-round structural and electronic control within $\mathrm{MoS}_{2}$ to enhance the HER performance has not been reported before.

Herein, we present a multiscale structural and electronic control of $\mathrm{MoS}_{2}$ foam for highly efficient HER process: (i) the macro-scale: a uniform mesoporous $\mathrm{MoS}_{2}$ foam (mPF-MoS average pore size $\sim 30 \mathrm{~nm})$ facilitate the transport of $\mathrm{H}_{3} \mathrm{O}^{+}$and $\mathrm{H}_{2}$, and increases the accessibility of $\mathrm{MoS}_{2}$ surface; (ii) the nanoscale: oriented vertical growth of $\mathrm{MoS}_{2}$ nanosheets around the mesopores increase the number of edges as the active sites; (iii) the atomic-scale: further chemical doping with transition metal Co atoms into the mPF- $\mathrm{MoS}_{2}$ framework enhance the intrinsic HER activity (mPF-Co- $\mathrm{MoS}_{2}$ ). Such mPF-Co-MoS 2 electrocatalyst exhibits an excellent durability and a low overpotential of only $156 \mathrm{mV}$ at the current density of $10 \mathrm{~mA} \mathrm{~cm}^{-2}$, comparable to the most active $\mathrm{MoS}_{2}$-based HER electrocatalysts in acidic medium (Supplementary Table 1). Furthermore, the density functional theory (DFT) calculations confirmed the experimental results that an appropriate Co doping content can greatly promote the HER activity of $\mathrm{MoS}_{2}$. The strategies, introduced in the present work, may open new opportunities for the rational design of $\mathrm{MoS}_{2}$ through a multiscale structural and electronic control to strengthen the electrocatalytic HER and other energyrelated process, and possibly for the structural control of other $2 \mathrm{D}$ materials.

\section{Results}

Synthesis of mesoporous $\mathrm{MoS}_{2}$ foam. The uniform mesoporous $\mathrm{MoS}_{2}$ foam (mPF-MoS ${ }_{2}$ was prepared with the synthetic procedure illustrated in Fig. 1. First, $\left(\mathrm{NH}_{4}\right)_{6} \mathrm{Mo}_{7} \mathrm{O}_{24}$ molecules were homogeneously adsorbed onto the colloidal $\mathrm{SiO}_{2}$ nanospheres via a wet impregnation method. Then, the direct chemical reaction with $\mathrm{CS}_{2}$ on $\mathrm{SiO}_{2}$ surface was conducted to convert Mo precursors into small $\mathrm{MoS}_{2}$ domains. Because of the induction of the monodisperse $\mathrm{SiO}_{2}$ nanospheres template, these small

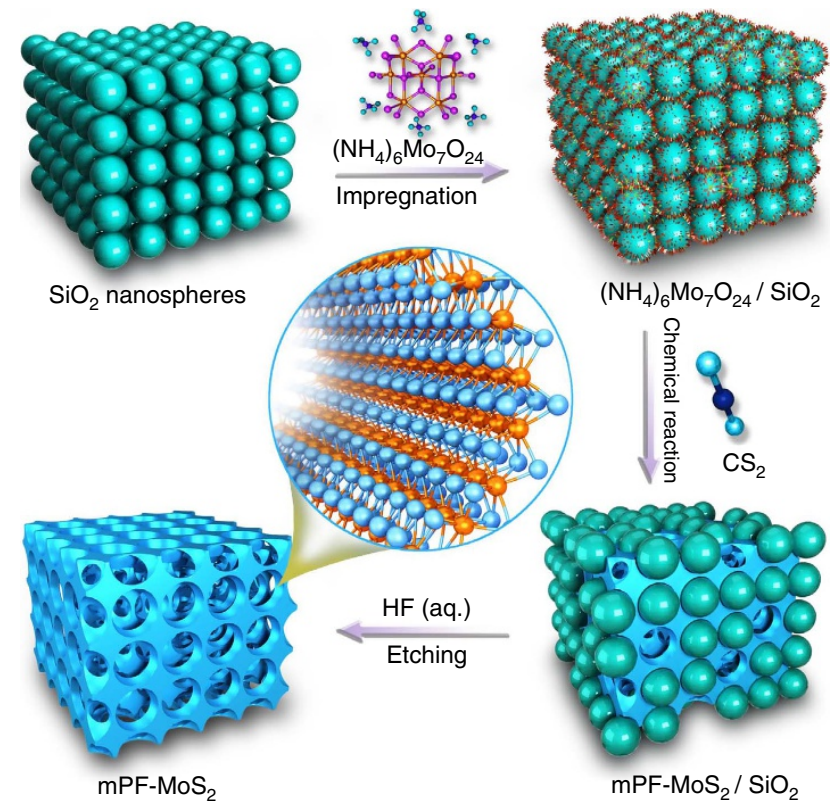

Figure 1 | Schematic illustration of the fabrication of mesoporous $\mathrm{MoS}_{\mathbf{2}}$ foam. The direct chemical synthesis was adopted with the $\left(\mathrm{NH}_{4}\right)_{6} \mathrm{Mo}_{7} \mathrm{O}_{24}$ and $\mathrm{CS}_{2}$ as precursors, assisted by the colloidal $\mathrm{SiO}_{2}$ nanospheres.

domains would further self-assembly into vertically aligned $\mathrm{MoS}_{2}$ layers around the $\mathrm{SiO}_{2}$ nanospheres. Finally, the mPF-MoS 2 can be obtained via etching the $\mathrm{SiO}_{2}$ template with $\mathrm{HF}$ solution. Note that the etching process will not influence the structure of $\mathrm{MoS}_{2}$ because the MoS 2 can not be dissolved by HF solution (Supplementary Fig. 1).

Structural analysis of mesoporous $\mathrm{MoS}_{2}$ foam. The scanning electron microscopy (SEM) and transmission electron microscopy (TEM) as well as high-angle annular dark field-scanning transmission electron microscopy (HAADF-STEM) show that the $\mathrm{mPF}-\mathrm{MoS}_{2}$ possessed abundant spherical voids derived from the residual spaces after the removal of $\mathrm{SiO}_{2}$ nanospheres, leading to a uniform porous framework (Fig. 2a-d, Supplementary Figs 2 and 3a). Note that these uniform nanopores are interconnected throughout the entire 3D MoS 2 foam at different orientations by $3 \mathrm{D}$ tomography (Supplementary Figs 4 and 5, Supplementary Movies 1 and 2), which facilitates the mass transport and accessibility of active sites during the catalytic process. The energy-dispersive X-ray (EDX) maps exhibits that the Mo and S elements were distributed homogeneously in the porous framework (Fig. 2e). The $\mathrm{N}_{2}$ adsorption-desorption isotherms indicate the presence of mesopores with a narrow pore size distribution at $\sim 24 \mathrm{~nm}$ (Fig. 2f).

The high resolution (HR) TEM image shows a typical interlayer distance of $0.62 \mathrm{~nm}$ corresponding to the (002) plane of $\mathrm{MoS}_{2}$ (Fig. $2 \mathrm{~g}$ ), and the hexagonal $2 \mathrm{H}-\mathrm{MoS}_{2}$ crystal characteristics could also be gain from the X-ray diffraction (XRD) pattern (Fig. 2h). Remarkably, the $\mathrm{MoS}_{2}$ layers were almost vertically aligned around the mesopores with a large fraction of exposed edge sites (Fig. $2 \mathrm{~g}$ and Supplementary Fig. 3b). Compared with random-oriented $\mathrm{MoS}_{2}$ nanosheet $\left(\mathrm{rNS}-\mathrm{MoS}_{2}\right)$ sample prepared without $\mathrm{SiO}_{2}$ template, the mPF-MoS 2 showed no obvious difference in the XRD patterns (Fig. 2h), Raman spectra (Supplementary Fig. 6), X-ray photoelectron spectroscopy (XPS) (Supplementary Fig. 7) and X-ray absorption near-edge structure (XANES) spectra (inset of Fig. 2i). But according to the HRTEM images comparison 

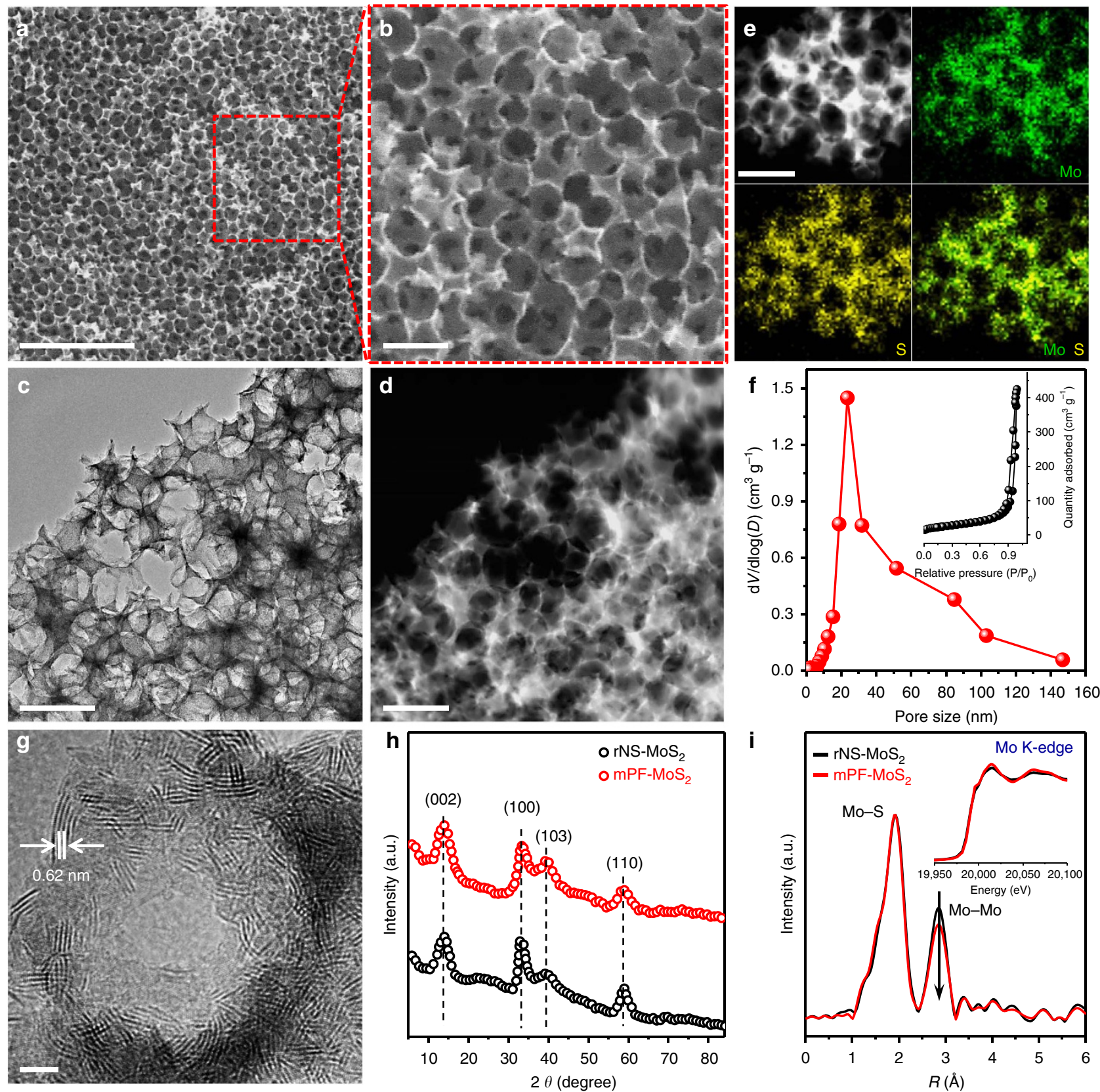

Figure 2 | Morphology and structural characterizations of mesoporous $\mathbf{M o S}_{\mathbf{2}}$ foam. (a,b) SEM images of mPF-MoS 2 . (c,d) TEM image and corresponding HAADF-STEM image of ${\mathrm{mPF}-M o S_{2}}_{2}$ at the same position. (e) HAADF-STEM image and corresponding EDX maps of mPF-MoS 2 . (f) Pore size distribution

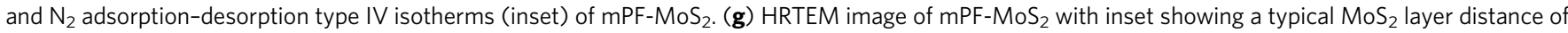

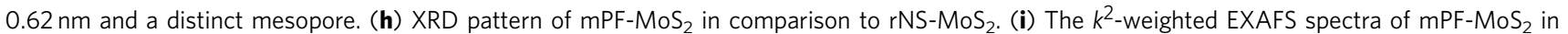

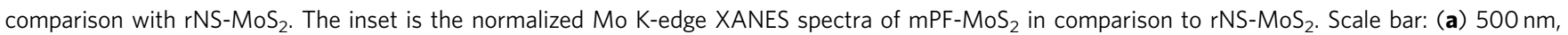
(b-e) $100 \mathrm{~nm},(\mathbf{g}) 5 \mathrm{~nm}$

(Supplementary Fig. 3), the mPF-MoS 2 with vertical aligned layer and smaller lateral size possessed much more exposed edge sites. In addition, the extended X-ray absorption fine structure (EXAFS) spectra (Fig. 2i) exhibited that $\mathrm{mPF}-\mathrm{MoS}_{2}$ had less Mo-Mo coordination than rNS-MoS ${ }_{2}$, also confirming the $\mathrm{mPF}-\mathrm{MoS}_{2}$ possessed more edge sites. This should increase the catalytic activity of $\mathrm{mPF}-\mathrm{MoS}_{2}$ significantly.

Electrocatalytic performance of mesoporous $\mathrm{MoS}_{2}$ foam. A typical three-electrode setup in $0.5 \mathrm{M} \mathrm{H}_{2} \mathrm{SO}_{4}$ electrolyte was adopted to conduct the electrocatalytic measurements. Bulk $\mathrm{MoS}_{2}$ shows a poor HER activity with only a minor improvement observed for rNS-MoS 2 (Fig. 3a). From Fig. 3b, one could see that compared with rNS-MoS 2 , the required overpotential to drive a HER current density of 10,20 and $50 \mathrm{~mA} \mathrm{~cm}^{-2}$ within mPF-MoS 2 reduced 195, 219 and $262 \mathrm{mV}$, respectively. Particularly, the overpotential at a current density of $10 \mathrm{~mA} \mathrm{~cm}^{-2}$ for $\mathrm{mPF}-\mathrm{MoS}_{2}$ is $210 \mathrm{mV}$, which is superior to the reported dense vertically aligned $\mathrm{MoS}_{2}$ film ${ }^{30}$. Furthermore, the $\mathrm{mPF}^{-\mathrm{MoS}_{2}}$ showed a long-term stable performance within the accelerated degradation measurements by 5,000 cyclic voltammetric (CV) sweeps (Fig. 3c), which also indicated $m P F-M_{2}$ is a good non-precious alternative for HER electrocatalyst. 

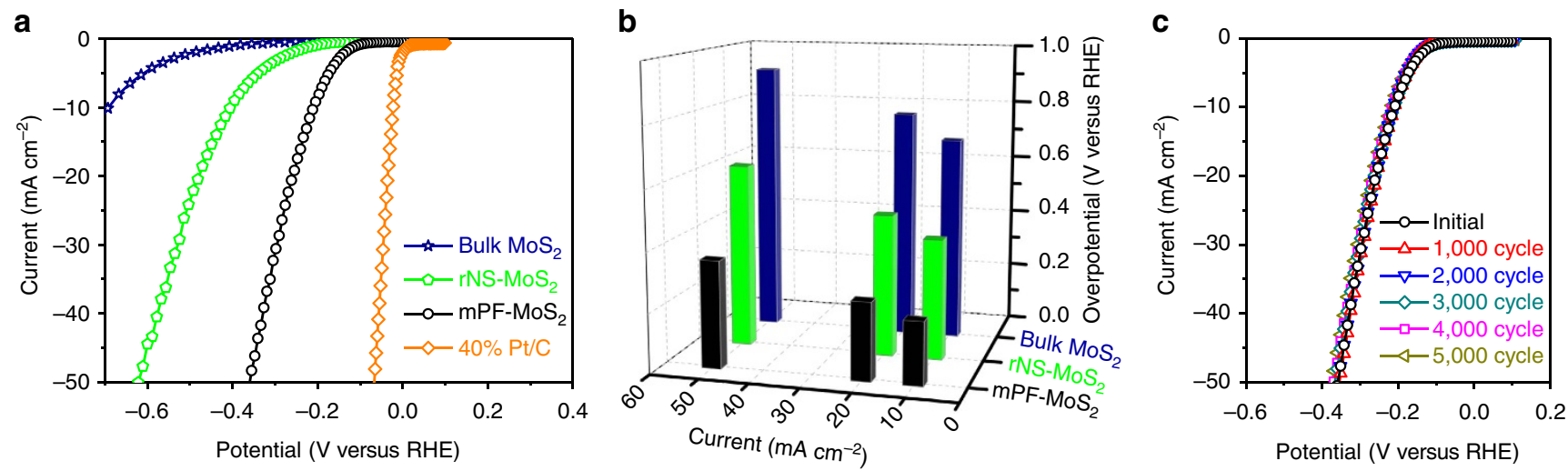

Figure 3 | Electrocatalytic HER performance of mesoporous $\mathbf{M o S}_{\mathbf{2}}$ foam. (a) HER polarization curves for mPF-MoS 2 in comparison with bulk MoS rNS-MoS 2 and $40 \% \mathrm{Pt} / \mathrm{C}$. (b) Overpotential at current density of 10,20 and $50 \mathrm{~mA} \mathrm{~cm}{ }^{-2}$ for mPF-MoS compared with rNS-MoS $_{2}$ and bulk MoS . (c) Durability measurement of $\mathrm{mPF}_{-} \mathrm{MoS}_{2}$. The polarization curves were recorded initially and after every 1,000 sweeps between -0.1 and $+0.5 \mathrm{~V}$ (versus RHE) at $100 \mathrm{mVs}^{-1}$. All the $\mathrm{HER}$ measurements were conducted in an Ar-saturated $0.5 \mathrm{M} \mathrm{H}_{2} \mathrm{SO}_{4}$ electrolyte at $25^{\circ} \mathrm{C}$.

It is usually considered that only the edge sites of pure $\mathrm{MoS}_{2}$ own the HER activity, while the basal plane is catalytically inert $^{14,15}$. Therefore, the $\mathrm{mPF}-\mathrm{MoS}_{2}$, possessing rich vertical edge sites, is expected to be more catalytically active. Moreover, numerous mesopores in $\mathrm{mPF}-\mathrm{MoS}_{2}$ facilitate the mass transport. Meanwhile, according to the contact angle measurements (Supplementary Fig. 8), the $\mathrm{mPF}-\mathrm{MoS}_{2}\left(23^{\circ}\right)$ become more hydrophilic relative to the $\mathrm{rNS}-\mathrm{MoS}_{2}\left(32^{\circ}\right)$ and bulk $\mathrm{MoS}_{2}\left(105^{\circ}\right)$, leading to the more easy accessibility of reactants on active sites for the mPF- $\mathrm{MoS}_{2}$ catalyst. In addition, the massive mesopores with curved surface in $\mathrm{MoS}_{2} 2 \mathrm{D}$ plane may induce the strain, which can further increase the electrocatalytic activity referring to the literatures ${ }^{19,33}$.

Chemical doping of mesoporous $\mathrm{MoS}_{2}$ foam. Doping of different transition metal atoms into the $\mathrm{MoS}_{2}$ matrix can enhance the intrinsic activity of its in-plane $S$ atoms ${ }^{32}$. Here, we introduced Co atoms into the $\mathrm{mPF}-\mathrm{MoS}_{2}$ framework by in situ adding Co precursors within the impregnation procedure (see the experimental section for details), yielding a Co-doped mesoporous $\mathrm{MoS}_{2}$ foam (mPF-Co-MoS $)$. As shown in the SEM (Supplementary Fig. 9), TEM (Supplementary Fig. 10a) and HAADF-STEM (Fig. 4a) images, the mesoporous $\mathrm{MoS}_{2}$ foam has been well retained after Co doping, with $\mathrm{MoS}_{2}$ flakes still assembling as vertically aligned layers around the mesopores (Supplementary Fig. 10b). The Co dopants bring indiscernible chemical state variation of the $\mathrm{MoS}_{2}$ framework according to the XPS spectra (Supplementary Fig. 11) and Mo K-edge XANES spectra (Supplementary Fig. 12). No Co-containing nanoparticles were observed from TEM images, consistent with the EDX maps showing the homogeneous distribution of Co, Mo and S elements over the entire mesoporous framework (Fig. 4a).

Co doping contents within the mesoporous $\mathrm{MoS}_{2}$ foam can be easily modulated by varying the amount of Co precursors, resulting in a series of $\mathrm{mPF}-\mathrm{Co}-\mathrm{MoS}_{2}$-x samples (x represents the Co doping contents in wt.\%). As shown in the Co K-edge XANES spectra (Fig. 4b) and EXAFS spectra (Fig. 4c), all Co atoms in different mesoporous $\mathrm{MoS}_{2}$ foam possess the valence and the Co-S bonds are distinguished from those in commercial CoS crystal. This indicates that Co atoms are covalently doped into the $\mathrm{MoS}_{2} 2 \mathrm{D}$ plane rather than being adsorbed on the surface. This finding is also confirmed by the Mo K-edge EXAFS spectra (Fig. 4d) showing a decrease of Mo-Mo coordination caused by the substituted-doping of Co atoms within the $\mathrm{MoS}_{2} 2 \mathrm{D}$ plane. In addition, the decrease of Mo-Mo coordination accompanied with the increase of Co doping contents (Fig. 4d) was also consistent with the stepwise red shift of $E_{2 g}^{1}$ and $A_{1 g}$ modes in Raman spectra (Fig. 4e) resulted from the progressively increased Co dopants in $\mathrm{MoS}_{2} 2 \mathrm{D}$ plane to soften the Mo-S related modes and decrease their vibration frequency ${ }^{34}$. Nevertheless, the $\mathrm{E}_{2 \mathrm{~g}}^{1}$ and $\mathrm{A}_{1 \mathrm{~g}}$ modes of $\mathrm{MoS}_{2}$ will change significantly when the Co doping contents exceeded $16.7 \%$ (Fig. 4e), suggesting a structural variation in the mesoporous $\mathrm{MoS}_{2}$ foam. This correlates with the XRD patterns showing that the crystal structure of $\mathrm{MoS}_{2}$ was well maintained with no other phases appearing after Co doping, until the Co doping contents were $21.1 \%$ or more (Fig. 4f). Meanwhile, distinct change in pore structure of $\mathrm{mPF}-\mathrm{Co}-\mathrm{MoS}_{2}$ samples appeared when the Co doping contents exceeds $16.7 \%$ (Supplementary Fig. 13). Note that mesoporous $\mathrm{MoS}_{2}$ foam with different Co doping contents showed no obvious difference in the contact angle measurements (Supplementary Fig. 14). The above analyses indicated that there is an optimum doping content (16.7\% from our experience) which will provide significant Co contents but still preserving the $\mathrm{mPF}-\mathrm{Co}-\mathrm{MoS}_{2}$ integrated mesoporous vertically aligned framework.

Effect of Co dopant on electrocatalytic performance. In view of the additional atomic-scale modulation in mesoporous $\mathrm{MoS}_{2}$ foam, a further enhanced HER process was expected. Thus, mPF-Co- $\mathrm{MoS}_{2}-3.4$ exhibited a distinctly enhanced activity, reducing the overpotential of 26 and $53 \mathrm{mV}$ at the current

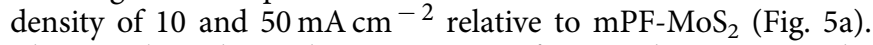
The sample with Co doping content of $16.7 \%$ demonstrates the optimum activity (Fig. 5b). The volcano-shaped relationship between HER activity and Co doping contents confirmed our finding that there is an optimum Co doping level which, from one hand enhances the intrinsic catalytic activity of $\mathrm{mPF}-\mathrm{MoS}_{2}$ and at the same time maintains the inherent framework within mesoporous $\mathrm{MoS}_{2}$ foam. Remarkably, the mPF-Co-MoS -16.7 showed a high HER activity with the overpotential at a current density of $10 \mathrm{~mA} \mathrm{~cm}^{-2}$ of only $156 \mathrm{mV}$ (Fig. 5a), comparable to the most active $\mathrm{MoS}_{2}$-based non-precious HER electrocatalysts in acidic medium (Supplementary Table 1). Moreover, mPF-Co- $\mathrm{MoS}_{2}-16.7$ also showed a long-term stable performance even after 5,000 CV sweeps within the accelerated degradation measurements (Fig. 5c). Tafel plots showed that mPF-Co-MoS -16.7 with a Tafel slope value of $74 \mathrm{mV} \mathrm{dec}^{-1}$ and mPF-MoS $2\left(90 \mathrm{mV} \mathrm{dec}^{-1}\right)$ followed a similar reaction process via 
a
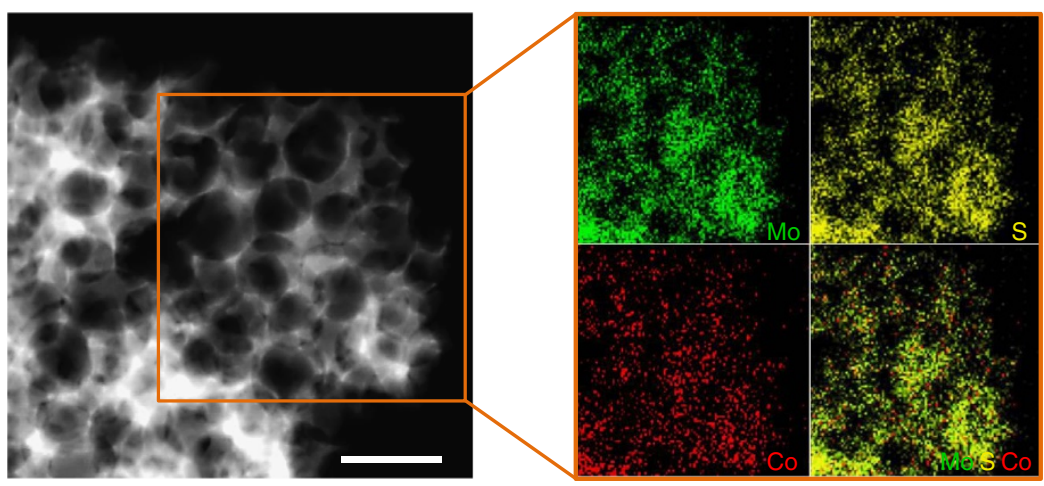

C

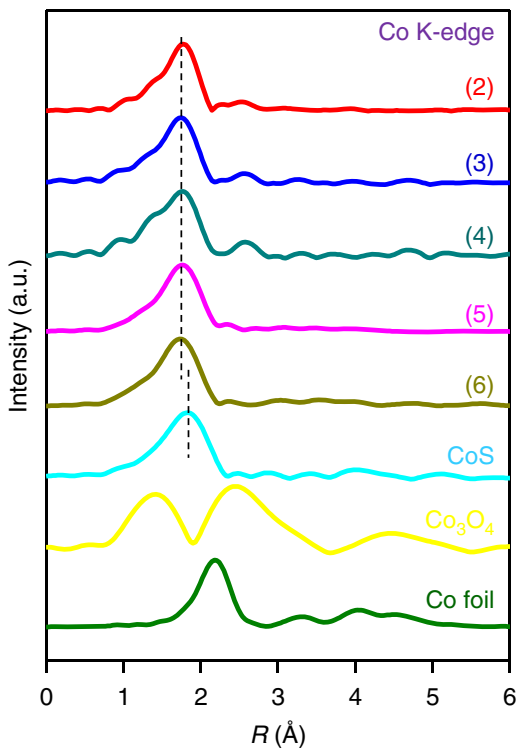

d

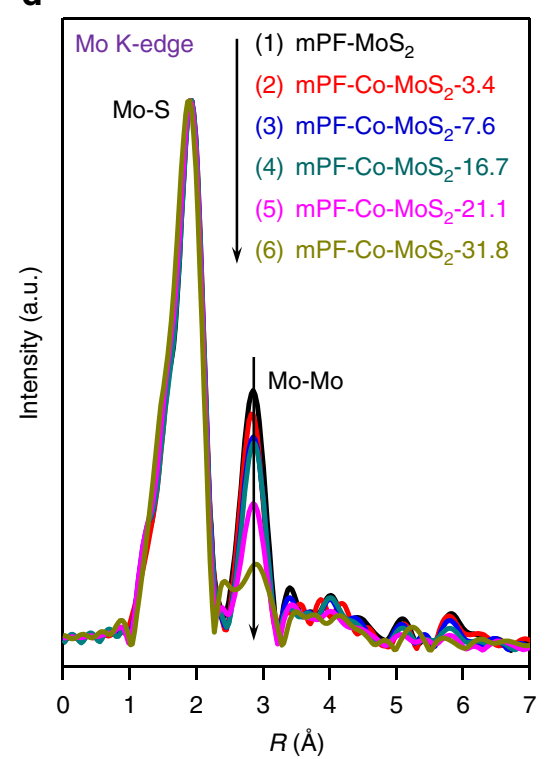

b

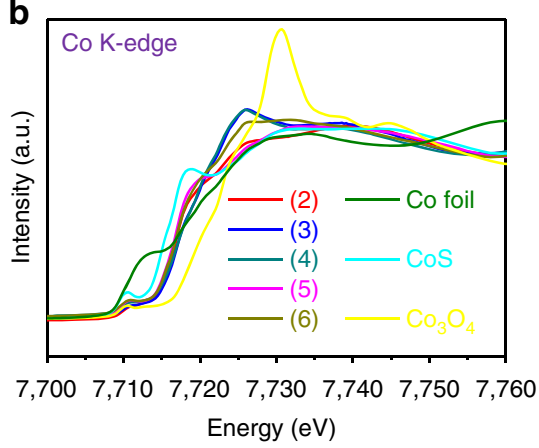

e
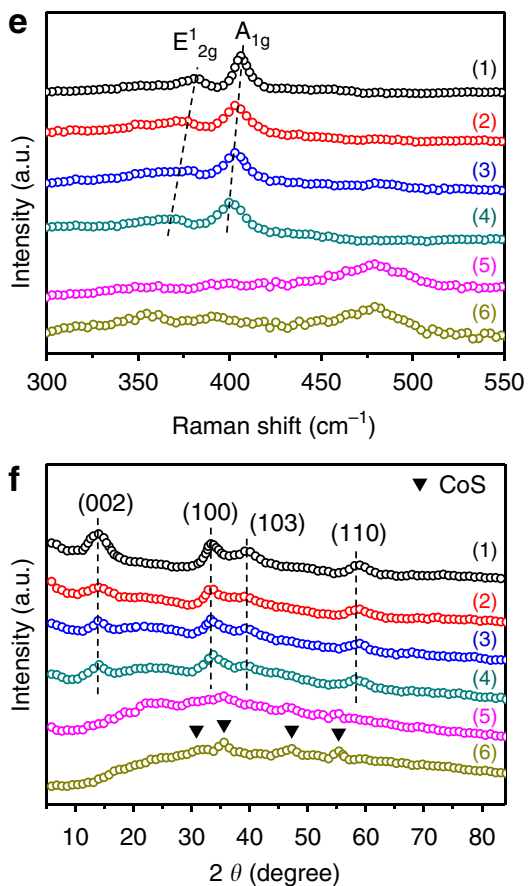

Figure 4 | Structural and electronic properties of various Co-doped mesoporous $\mathbf{M o S}_{\mathbf{2}}$ foam. (a) HAADF-STEM image and corresponding EDX maps with orange rectangle in HAADF-STEM image of mPF-Co-MoS 2 -16.7. Scale bar, $100 \mathrm{~nm}$. (b) Co K-edge XANES spectra of a series of mPF-Co-MoS 2 samples in comparison to $\mathrm{Co}$ foil, $\mathrm{CoS}$, and $\mathrm{Co}_{3} \mathrm{O}_{4}$, respectively. (c) Co K-edge $k^{2}$-weighted EXAFS spectra of a series of $\mathrm{mPF}$-Co-MoS 2 samples in comparison with $\mathrm{CoS}, \mathrm{Co}_{3} \mathrm{O}_{4}$ and $\mathrm{Co}$ foil, respectively. (d) Mo K-edge $k^{2}$-weighted EXAFS spectra of various mPF-Co-MoS ${ }_{2}$ samples compared with mPF$\mathrm{MoS}_{2}$. (e) Raman spectra of different mPF-Co-MoS samples in comparison to mPF-MoS 2 . (f) XRD patterns of a series of mPF-Co-MoS 2 samples in comparison with mPF-MoS . The numbers (1), (2), (3), (4), (5) and (6) represent mPF-MoS 2 and mPF-Co-MoS 2 with the Co doping contents of 3.4, 7.6, 16.7, 21.1 and $31.8 \%$, respectively.

the Volmer-Heyrovsky mechanism ${ }^{35-37}$, deviating from the Pt/C electrocatalyst $\left(30 \mathrm{mV} \mathrm{dec}^{-1}\right)$ via the Volmer-Tafel mechanism (Fig. 5d). These results demonstrated that the Co doping content will significantly affect the activity modulation of $\mathrm{MoS}_{2}$, and a moderate value can maximally promote the multiscale structural and electronic control in mesoporous $\mathrm{MoS}_{2}$ foam for the HER activity optimization.

Theoretical studies of Co doping effect. DFT calculations were carried out to gain further insights into the influence of different Co doping contents within the basal plane of $\mathrm{MoS}_{2}$ on the HER activity. The hydrogen adsorption free energy $\left(\Delta G_{\mathrm{H}}\right)$ is a widely accepted descriptor of HER activity for various catalytic materials, where the optimal value of $\Delta G_{\mathrm{H}}$ is around zero $(\sim 0) \mathrm{eV}$ to compromise the reaction barriers and achieve the best HER activity ${ }^{38,39}$. For the basal plane of pristine $\mathrm{MoS}_{2}$, the $\Delta G_{\mathrm{H}}$ is $\sim 2 \mathrm{eV}$, far away from the optimal value. With Co atoms introduced into the $\mathrm{MoS}_{2}$ in-plane, taking the coverage $\left(\theta_{\mathrm{H}}\right)$ of
$1 / 4$ monolayer (ML) as an example, the $\Delta G_{\mathrm{H}}$ decreased continuously and reached $\sim 0 \mathrm{eV}$ at the Co doping content of 13.3 wt.\% (atomic ratio of Co:Mo is 1:2), beyond which the $\Delta G_{\mathrm{H}}$ will depart away from the optimal values again (Fig. 6a). These simulations indicate that there indeed exists a moderate Co doping content to promote $\mathrm{MoS}_{2}$ to gain the optimal HER activity, confirming the experimental results.

To understand the origin of the increased HER activity with increased Co doping content, an analysis of the electronic properties has been made. First, the projected density of state of $S$ atoms show a significant increase in the electronic states of inplane S sites around Fermi level after Co atoms doping (Supplementary Fig. 16), resulting in the enhanced catalytic activity, in accordance with our previous study ${ }^{32}$. Furthermore, according to the molecular orbital theory, when $\mathrm{H}$ atom is absorbed on surface $\mathrm{S}$ atom, the combination of $\mathrm{H} 1 s$ orbital and S $3 p$ orbital will form a bonding orbital $(\sigma)$ and anti-bonding orbital $\left(\sigma^{\star}\right)$, where the degree of energy level matching between $\mathrm{H}$ atom and $\mathrm{S}$ atom determines the $\mathrm{H}-\mathrm{S}$ bonding strength (Fig. $6 \mathrm{~b}$ ). 

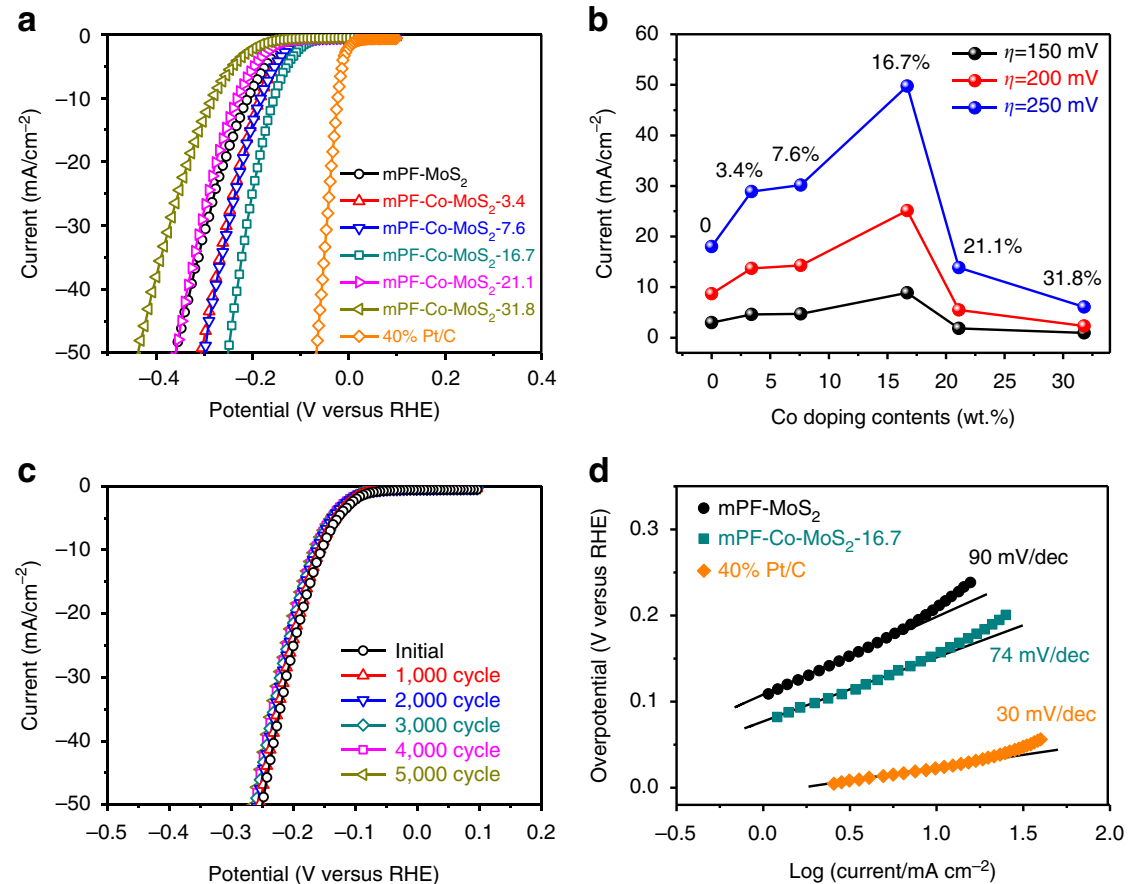

Figure 5 | Effect of Co doping on the HER performance of mesoporous $\mathbf{M o S}_{\mathbf{2}}$ foam. (a) HER polarization curves for mPF-Co-MoS 2 with different Co doping contents in comparison with $\mathrm{mPF}-\mathrm{MoS}_{2}$ and $40 \% \mathrm{Pt} / \mathrm{C}$. (b) Current densities at overpotential of 150, 200 and $250 \mathrm{mV}$ for mPF-Co-MoS 2 with

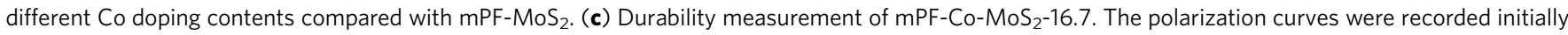

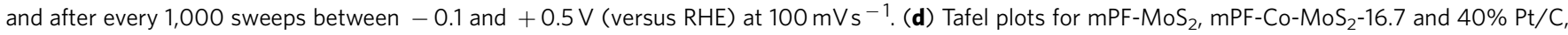
respectively. All the HER measurements were conducted in an Ar-saturated $0.5 \mathrm{M} \mathrm{H}_{2} \mathrm{SO}_{4}$ electrolyte at $25^{\circ} \mathrm{C}$.

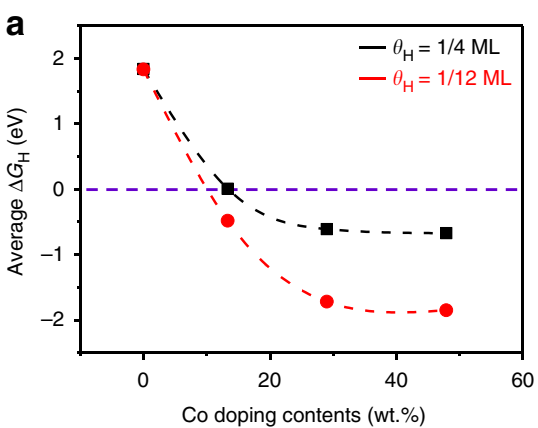

b

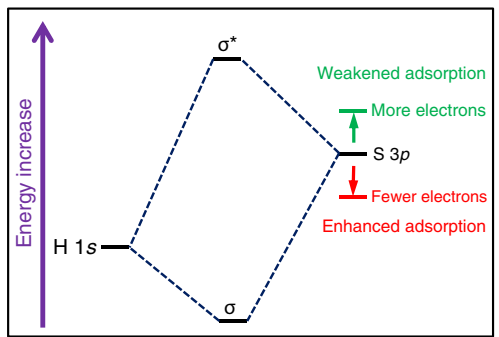

C
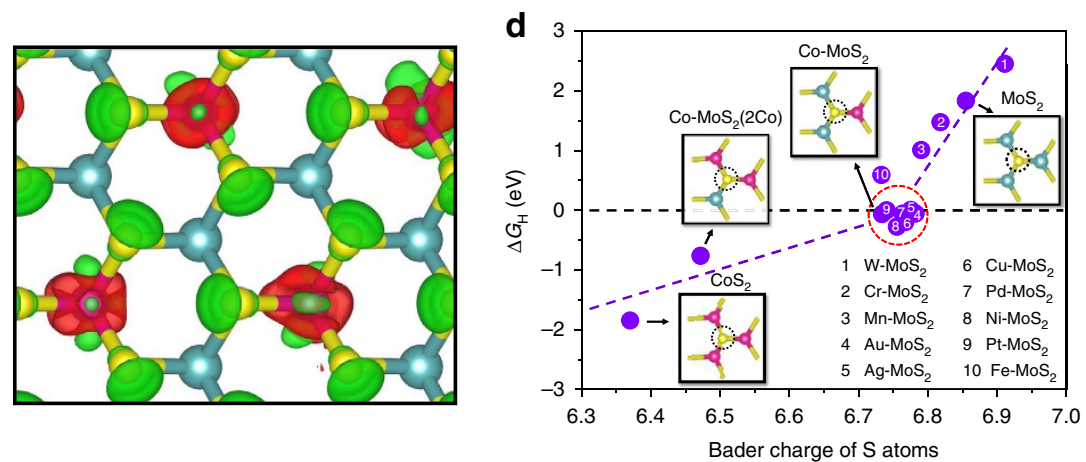

Figure 6 | Theoretical calculations for the effect of Co doping contents on HER of $\mathbf{M o S}_{\mathbf{2}}$. (a) Average $\Delta G_{H}$ on $\mathbf{S}$ atoms versus the Co doping contents, considering different coverage of $1 / 4 \mathrm{ML}$ and 1/12 ML. The corresponding optimized catalyst structures can be seen in Supplementary Fig. 15. (b) Schematic diagram of the bonding of $\mathrm{H}$ 1s orbital and $\mathrm{S} 3 p$ orbital (from $\mathrm{MoS}_{2}$ ), where depletion of electrons on $\mathrm{S}$ atoms will lower the orbital position and enhance the $\mathrm{H}-\mathrm{S}$ bond. (c) Differential charge density of Co-doped $\mathrm{MoS}_{2}$ (Co doping content of 13.3 wt.\%, Co:Mo atomic ratio of 1:2). Red and green contours represent electron accumulation and depletion, respectively. The isosurface level is set to be $0.11 \mathrm{e} / \mathrm{Bohr}^{3}$. (d) $\Delta G_{H}$ on $\mathrm{S}$ atoms versus the Bader charge of $\mathrm{S}$ atoms for different structures, with the detailed data for each point shown in Supplementary Table 2. The insets are the atomic configurations of one $S$ atom bonding with three Co, two Co and one Mo, one Co and two Mo, as well as three Mo atoms, respectively. Green balls: Mo; yellow balls: S; pink balls: Co. 
Because of the very high energy level of $\mathrm{S} 3 p$ orbital relative to $\mathrm{H} 1 s$ orbital, the $\mathrm{H}$ adsorption on basal plane of pristine $\mathrm{MoS}_{2}$ is too weak $\left(\Delta G_{\mathrm{H}}=\sim 2 \mathrm{eV}\right)$, leading to a poor HER performance of $\mathrm{MoS}_{2}$ for HER. When doping another metal atom such as Co into the $\mathrm{MoS}_{2}$ in-plane, the electron number on $\mathrm{S}$ atom will decrease (Fig. 6c) to offset the energy level mismatching for enhancing the $\mathrm{H}$ adsorption and HER activity. Different metal atoms own the different capability to modify the electron density on $\mathrm{S}$ atoms (see Fig. 6d and Supplementary Table 2 for detailed data), and the metal dopants that tune the Bader charge of $\mathrm{S}$ atoms into the range of $\sim 6.73$ to $\sim 6.78$ will lead to a moderate $\Delta G_{\mathrm{H}}$ (Fig. $6 \mathrm{~d}$ ) and high HER activity (Supplementary Fig. 17). Among them, the Co atom is indeed a good regulator to bring the $\Delta G_{\mathrm{H}}$ get $\sim 0 \mathrm{eV}$. Nevertheless, further increasing Co doping contents will cause the excessive decrease of electron on $\mathrm{S}$ atoms to make the interaction between $\mathrm{H}$ atoms and $\mathrm{S}$ atoms too strong (Fig. 6d). In addition, high Co doping contents can also lead to less stable of $\mathrm{MoS}_{2}$ surface according to the surface energy $(\gamma)$ calculations (Supplementary Fig. 18), which is also harmful to the HER activity.

\section{Discussion}

In summary, we introduce a multiscale structural and electronic control of $\mathrm{MoS}_{2}$ strategy to achieve the high-efficient HER electrocatalysis. First, a uniform mesoporous $\mathrm{MoS}_{2}$ foam $\left(\mathrm{mPF}-\mathrm{MoS}_{2}\right)$ was fabricated with a significantly enhanced HER performance compared with that of random-oriented $\mathrm{MoS}_{2}$ nanosheet $\left(\mathrm{rNS}-\mathrm{MoS}_{2}\right)$. It originates from the macro-scale modulation fabricating massive mesopores to gain the sufficient transport of $\mathrm{H}_{3} \mathrm{O}^{+}$and $\mathrm{H}_{2}$, the favourable accessibility of $\mathrm{MoS}_{2}$ surface and the strain-induced promotion, as well as the nano-scale modulation with vertically aligned layers to provide abundant active edge sites. Second, chemical doping was introduced to add further atomic-scale engineering in the mesoporous $\mathrm{MoS}_{2}$ foam for the intrinsic activity increase. The optimum Co-doped mesoporous $\mathrm{MoS}_{2}$ foam (mPF-Co-MoS 2 ) with a Co content of $16.7 \%$ showed a further distinct enhancement of HER activity, which possessed a long-term durability with more than 5,000 recycles and an overpotential of only $156 \mathrm{mV}$ at the current density of $10 \mathrm{~mA} \mathrm{~cm}^{-2}$, comparable to the most active $\mathrm{MoS}_{2}$-based electrocatalysts in acidic medium. DFT calculations confirmed the experimental results that a moderate Co doping content can modulate the $\mathrm{H}$ adsorption on $\mathrm{MoS}_{2}$ to a suitable degree and simultaneously maintain the structure stability to promote the HER activity reach optimum value. The findings in the present work pave a rational pathway to strengthen the electrocatalytic HER performance of $\mathrm{MoS}_{2}$ via the multiscale structural and electronic control, and the involved concept and strategy can be extended to other energy-related process or other $2 \mathrm{D}$ materials.

\footnotetext{
Methods

Materials synthesis. The $\mathrm{mPF}-\mathrm{MoS}_{2}$ was synthesized through a direct chemical synthesis method. First, $400 \mathrm{mg}\left(\mathrm{NH}_{4}\right)_{6} \mathrm{Mo}_{7} \mathrm{O}_{24} \cdot 4 \mathrm{H}_{2} \mathrm{O}$ and 5,333.4 $\mathrm{mg} \mathrm{SiO}_{2}$ colloidal disperse ( $30 \mathrm{wt} . \% \mathrm{SiO}_{2}$ in ethylene glycol, Alfa Aesar) were dispersed in $20 \mathrm{ml}$ deionized water, followed by stirring under room temperature to remove the solvent and drying under $80^{\circ} \mathrm{C}$. Then, the gained solid and $10 \mathrm{ml} \mathrm{CS}$ were transferred into a $40 \mathrm{ml}$ stainless steel autoclave under $\mathrm{Ar}$ and maintained at $400^{\circ} \mathrm{C}$ for $4 \mathrm{~h}$. The final product was treated with HF (aq.) under room temperature for $5 \mathrm{~h}$, followed by washing with water and absolute ethanol for several times and drying at $80^{\circ} \mathrm{C}$. For comparison, the rNS- $\mathrm{MoS}_{2}$ was synthesized by using $900 \mathrm{mg}$ $\left(\mathrm{NH}_{4}\right)_{6} \mathrm{Mo}_{7} \mathrm{O}_{24} \cdot 4 \mathrm{H}_{2} \mathrm{O}$ dissolved in $20 \mathrm{ml}$ deionized water and $10 \mathrm{ml} \mathrm{CS}_{2}$ conducted within the same chemical reaction as the $\mathrm{mPF}-\mathrm{MoS}_{2}$ without using $\mathrm{SiO}_{2}$ template. The series of mPF-Co- $\mathrm{MoS}_{2}$ samples were synthesized by using $400 \mathrm{mg}$ $\left(\mathrm{NH}_{4}\right)_{6} \mathrm{Mo}_{7} \mathrm{O}_{24} \cdot 4 \mathrm{H}_{2} \mathrm{O}$, specified amount of $\mathrm{Co}\left(\mathrm{NO}_{3}\right)_{2} \cdot 6 \mathrm{H}_{2} \mathrm{O}$ and 5,333.4 mg $\mathrm{SiO}_{2}$ colloidal dispers to gain the impregnated solid, and then with $10 \mathrm{ml} \mathrm{CS}_{2}$ to proceed
} within the same process as the mPF-MoS $\mathrm{M}_{2}$. The Co doping contents in final
mPF-Co- $\mathrm{MoS}_{2}$ samples were measured by inductively coupled plasma atomic emission spectroscopy.

Materials characterization. SEM was conducted on Hitachi S4800 operated at $20 \mathrm{kV}$. TEM, HAADF-STEM and EDX mapping were carried out on a FEI Tecnai 30 microscope and a 20 microscope operated at an accelerating voltage of 300 and $200 \mathrm{kV}$, respectively. The 3D tomography in the STEM mode was carried out on the FEI Talos F $200 \times$ microscope operated at $200 \mathrm{kV} . \mathrm{N}_{2}$ adsorptiondesorption was measured with a Micromeritics Tristar 3020 Surface Area and Porosimetry analyzer. XRD measurements were conducted on a Rigaku Ultima IV diffractometer with $\mathrm{Cu} \mathrm{K} \alpha$ radiation at $35 \mathrm{kV}$ and $15 \mathrm{~mA}$. XANES and EXAFS were measured at the BL14W1 beamline of the Shanghai Synchrotron Radiation Facility (SSRF). Raman spectroscopy was performed on a Renishaw inVia Raman microscope with a $532 \mathrm{~nm}$ excitation laser at a power of $0.29 \mathrm{~mW}$. XPS measurements were carried out on an Omicron XPS System used $\mathrm{Al} \mathrm{K \alpha}$ X-rays as the excitation source with a voltage of $15 \mathrm{kV}$ and power of $300 \mathrm{~W}$. Contact angle of water solution droplet on the surface of catalyst layer were conducted on SDC-100 contact angle measurement instrument (Shengding Precision Instrument Co., Ltd., China) at room temperature. Inductively coupled plasma atomic emission spectroscopy was carried out in Varian AA240z graphite furnace atomic absorption spectrometer.

Electrochemical measurements. HER polarization curve tests were conducted on a Princeton Parstat MC potentiostat/galvanostat with a three-electrode electrochemical cell equipped with a gas flow controlling system. Graphite rod was used as the counter electrode and $\mathrm{Ag} / \mathrm{AgCl}$ (saturated $\mathrm{KCl}$-filled) as the reference electrode. A glassy carbon rotating disk electrode with a diameter of $5 \mathrm{~mm}$ covered by a thin catalyst film was used as the working electrode. Typically, $4 \mathrm{mg}$ catalyst was suspended in $1 \mathrm{ml}$ ethanol with $20 \mu \mathrm{l}$ Nafion solution ( $5 \mathrm{wt} . \%$, Du Pont) to form a homogeneous ink assisted by ultrasound. Then $25 \mu \mathrm{l}$ of the ink was spread onto the surface of glassy carbon by a micropipette and dried under room temperature. The final loading for the catalysts and $40 \% \mathrm{Pt} / \mathrm{C}$ electrocatalysts on work electrode is $0.5 \mathrm{mg} \mathrm{cm}^{-2}$. HER tests were conducted in an Ar-saturated $0.5 \mathrm{M} \mathrm{H}_{2} \mathrm{SO}_{4}$ electrolyte at $25^{\circ} \mathrm{C}$. The potential range was from 0 to $-1.0 \mathrm{~V}$ (versus $\mathrm{Ag} / \mathrm{AgCl}$ ) and the scan rate was $2 \mathrm{mV} \mathrm{s}^{-1}$. Before measurements, the samples were repeatedly swept from -0.4 to $0.3 \mathrm{~V}$ (versus $\mathrm{Ag} / \mathrm{AgCl}$ ) in the electrolyte until a steady voltammogram curve was obtained. All the final potentials have been calibrated with respect to a reversible hydrogen electrode (RHE).

DFT calculations. All theoretical calculations were performed using Vienna $a b$ initio simulation packages (VASP) ${ }^{40}$ with projector-augmented wave scheme ${ }^{41}$. The generalized gradient approximation with the Perdew-Burke-Ernzerhof $(\mathrm{PBE})^{42}$ functional was used for the exchange-correlation interaction. The plane wave cutoff was set to $400 \mathrm{eV}$. The convergence of total energy and forces were set to $1 \times 10^{-5} \mathrm{eV}$ and $0.05 \mathrm{eV} \AA^{-1}$, respectively. A periodically repeated single-layer $\mathrm{MoS}_{2}$ (a trilayer unit of S-Mo-S as a single layer ${ }^{43}$ ) crystal model with a $20 \AA$ vacuum space has been built for DFT calculations. The Brillouin zone was sampled by a $3 \times 3 \times 1 k$-point grid with the Monkhorst-Pack scheme ${ }^{44}$ for structural optimization and a $6 \times 6 \times 1 k$-point grid for electronic structure calculations. More details see the Supplementary Methods.

Data availability. The data that support the findings of this study are available from the corresponding authors on request.

\section{References}

1. Liu, Z. K., Lau, S. P. \& Yan, F. Functionalized graphene and other twodimensional materials for photovoltaic devices: device design and processing. Chem. Soc. Rev. 44, 5638-5679 (2015).

2. Wang, Q. H., Kalantar-Zadeh, K., Kis, A., Coleman, J. N. \& Strano, M. S Electronics and optoelectronics of two-dimensional transition metal dichalcogenides. Nat. Nanotechnol. 7, 699-712 (2012).

3. $\mathrm{Gu}, \mathrm{X}$. et al. A solution-processed hole extraction layer made from ultrathin $\mathrm{MoS}_{2}$ nanosheets for efficient organic solar cells. Adv. Energy Mater. 3, 1262-1268 (2013).

4. Xiang, Q. J., Yu, J. G. \& Jaroniec, M. Synergetic effect of $\mathrm{MoS}_{2}$ and graphene as cocatalysts for enhanced photocatalytic $\mathrm{H}_{2}$ production activity of $\mathrm{TiO}_{2}$ nanoparticles. J. Am. Chem. Soc. 134, 6575-6578 (2012).

5. Ding, Q. et al. Efficient photoelectrochemical hydrogen generation using heterostructures of $\mathrm{Si}$ and chemically exfoliated metallic $\mathrm{MoS}_{2}$. J. Am. Chem. Soc. 136, 8504-8507 (2014).

6. Chang, K. et al. $\mathrm{MoS}_{2}$ /graphene cocatalyst for efficient photocatalytic $\mathrm{H}_{2}$ evolution under visible light irradiation. ACS Nano 8, 7078-7087 (2014).

7. Stephenson, T., Li, Z., Olsen, B. \& Mitlin, D. Lithium ion battery applications of molybdenum disulfide $\left(\mathrm{MoS}_{2}\right)$ nanocomposites. Energy Environ. Sci. 7, 209-231 (2014).

8. Chhowalla, M. et al. The chemistry of two-dimensional layered transition metal dichalcogenide nanosheets. Nat. Chem. 5, 263-275 (2013). 
9. Zhu, C. B., Mu, X. K., van Aken, P. A., Yu, Y. \& Maier, J. Single-layered ultrasmall nanoplates of $\mathrm{MoS}_{2}$ embedded in carbon nanofibers with excellent electrochemical performance for lithium and sodium storage. Angew. Chem. Int. Ed. 53, 2152-2156 (2014).

10. Deng, D. H. et al. Catalysis with two-dimensional materials and their heterostructures. Nat. Nanotechnol. 11, 218-230 (2016).

11. Asadi, M. et al. Cathode based on molybdenum disulfide nanoflakes for lithium-oxygen batteries. ACS Nano 10, 2167-2175 (2016).

12. Yuwen, L. H. et al. General synthesis of noble metal (Au, Ag, Pd, Pt) nanocrystal modified $\mathrm{MoS}_{2}$ nanosheets and the enhanced catalytic activity of Pd- $\mathrm{MoS}_{2}$ for methanol oxidation. Nanoscale 6, 5762-5769 (2014).

13. Huang, H., Feng, X., Du, C. C., Wu, S. Y. \& Song, W. B. Incorporated oxygen in $\mathrm{MoS}_{2}$ ultrathin nanosheets for efficient ORR catalysis. J. Mater. Chem. A 3, 16050-16056 (2015)

14. Hinnemann, B. et al. Biornimetic hydrogen evolution: $\mathrm{MoS}_{2}$ nanoparticles as catalyst for hydrogen evolution. J. Am. Chem. Soc. 127, 5308-5309 (2005).

15. Jaramillo, T. F. et al. Identification of active edge sites for electrochemical $\mathrm{H}_{2}$ evolution from $\mathrm{MoS}_{2}$ nanocatalysts. Science 317, 100-102 (2007).

16. Li, Y. G. et al. $\mathrm{MoS}_{2}$ nanoparticles grown on graphene: an advanced catalyst for the hydrogen evolution reaction. J. Am. Chem. Soc. 133, 7296-7299 (2011).

17. Huang, X. et al. Solution-phase epitaxial growth of noble metal nanostructures on dispersible single-layer molybdenum disulfide nanosheets. Nat. Commun. 4, 1444 (2013).

18. Deng, J. et al. High-performance hydrogen evolution electrocatalysis by layercontrolled $\mathrm{MoS}_{2}$ nanosheets. RSC Adv. 4, 34733-34738 (2014).

19. Li, H. et al. Activating and optimizing $\mathrm{MoS}_{2}$ basal planes for hydrogen evolution through the formation of strained sulphur vacancies. Nat. Mater. 15, 48-53 (2016).

20. Lukowski, M. A. et al. Enhanced hydrogen evolution catalysis from chemically exfoliated metallic $\mathrm{MoS}_{2}$ nanosheets. J. Am. Chem. Soc. 135, 10274-10277 (2013).

21. Liao, L. et al. $\mathrm{MoS}_{2}$ formed on mesoporous graphene as a highly active catalyst for hydrogen evolution. Adv. Funct. Mater. 23, 5326-5333 (2013).

22. Lin, T. Q. et al. Nitrogen-doped mesoporous carbon of extraordinary capacitance for electrochemical energy storage. Science 350, 1508-1513 (2015).

23. Jiao, Y. C. et al. Highly ordered mesoporous few-layer graphene frameworks enabled by $\mathrm{Fe}_{3} \mathrm{O}_{4}$ nanocrystal superlattices. Angew. Chem. Int. Ed. 54, 5727-5731 (2015).

24. Wang, G. et al. Controlled synthesis of N-doped carbon nanospheres with tailored mesopores through self-assembly of colloidal silica. Angew. Chem. Int. Ed. 54, 15191-15196 (2015).

25. Liu, S. H. et al. Patterning two-dimensional free-standing surfaces with mesoporous conducting polymers. Nat. Commun. 6, 8817 (2015).

26. Fang, Y. et al. Growth of single-layered two-dimensional mesoporous polymer/ carbon films by self-assembly of monomicelles at the interfaces of various substrates. Angew. Chem. Int. Ed. 54, 8425-8429 (2015).

27. Kibsgaard, J., Chen, Z. B., Reinecke, B. N. \& Jaramillo, T. F. Engineering the surface structure of $\mathrm{MoS}_{2}$ to preferentially expose active edge sites for electrocatalysis. Nat. Mater. 11, 963-969 (2012).

28. Gao, M. R., Chan, M. K. Y. \& Sun, Y. G. Edge-terminated molybdenum disulfide with a 9.4-angstrom interlayer spacing for electrochemical hydrogen production. Nat. Commun. 6, 7493 (2015).

29. Xie, J. F. et al. Defect-rich $\mathrm{MoS}_{2}$ ultrathin nanosheets with additional active edge sites for enhanced electrocatalytic hydrogen evolution. Adv. Mater. 25, 5807-5813 (2013).

30. Kong, D. S. et al. Synthesis of $\mathrm{MoS}_{2}$ and $\mathrm{MoSe}_{2}$ films with vertically aligned layers. Nano Lett. 13, 1341-1347 (2013).

31. Ye, G. L. et al. Defects engineered monolayer $\mathrm{MoS}_{2}$ for improved hydrogen evolution reaction. Nano Lett. 16, 1097-1103 (2016).

32. Deng, J. et al. Triggering the electrocatalytic hydrogen evolution activity of the inert two-dimensional $\mathrm{MoS}_{2}$ surface via single-atom metal doping. Energy Environ. Sci. 8, 1594-1601 (2015).

33. Lee, J. H., Jang, W. S., Han, S. W. \& Baik, H. K. Efficient hydrogen evolution by mechanically strained $\mathrm{MoS}_{2}$ nanosheets. Langmuir. 30, 9866-9873 (2014).

34. Li, H. L. et al. Growth of alloy $\mathrm{MoS}_{2 \mathrm{x}} \mathrm{Se}_{2(1-\mathrm{x})}$ nanosheets with fully tunable chemical compositions and optical properties. J. Am. Chem. Soc. 136, 3756-3759 (2014).

35. Conway, B. E. \& Tilak, B. V. Interfacial processes involving electrocatalytic evolution and oxidation of $\mathrm{H}_{2}$, and the role of chemisorbed $\mathrm{H}$. Electrochim. Acta 47, 3571-3594 (2002).
36. Merki, D., Vrubel, H., Rovelli, L., Fierro, S. \& Hu, X. L. Fe, Co, and Ni ions promote the catalytic activity of amorphous molybdenum sulfide films for hydrogen evolution. Chem. Sci. 3, 2515-2525 (2012).

37. Bockris, J. O. M. \& Potter, E. C. The mechanism of the cathodic hydrogen evolution reaction. J. Electrochem. Soc. 99, 169-186 (1952).

38. Norskov, J. K. et al. Trends in the exchange current for hydrogen evolution. J. Electrochem. Soc. 152, J23-J26 (2005).

39. Greeley, J., Jaramillo, T. F., Bonde, J., Chorkendorff, I. B. \& Norskov, J. K. Computational high-throughput screening of electrocatalytic materials for hydrogen evolution. Nat. Mater. 5, 909-913 (2006).

40. Kresse, G. \& Furthmuller, J. Efficient iterative schemes for ab initio totalenergy calculations using a plane-wave basis set. Phys. Rev. B 54, 11169-11186 (1996).

41. Blochl, P. E. Projector augmented-wave method. Phys. Rev. B 50, 17953-17979 (1994).

42. Perdew, J. P., Burke, K. \& Ernzerhof, M. Generalized gradient approximation made simple. Phys. Rev. Lett. 77, 3865-3868 (1996).

43. Tsai, C., Abild-Pedersen, F. \& Norskov, J. K. Tuning the $\mathrm{MoS}_{2}$ edge-site activity for hydrogen evolution via support interactions. Nano Lett. 14, 1381-1387 (2014).

44. Monkhorst, H. J. \& Pack, J. D. Special points for Brillouin-zone integrations. Phys. Rev. B 13, 5188-5192 (1976).

\section{Acknowledgements}

We gratefully acknowledge the financial support from the Ministry of Science and Technology of China (No. 2016YFA0204100 and 2016YFA0200200), the National Natural Science Foundation of China (No. 21573220 and 21621063), the Key Research Program of Frontier Sciences of the Chinese Academy of Sciences (No. QYZDB-SSWJSC020), the strategic Priority Research Program of the Chinese Academy of Sciences (No. XDA09030100). We thank staff at the BL14W1 beamline of the Shanghai Synchrotron Radiation Facilities (SSRF) for assistance with the EXAFS and XANES measurements. We also acknowledge the computational resources from National Supercomputing Center in Shenzhen.

\section{Author contributions}

X.B. and D.D. conceived the project and designed the experiments. J.D. performed the materials synthesis, materials characterization and electrochemical measurements. H.L. conducted the DFT calculations. S.W. assisted with the materials characterization. D.D and M.C. performed the XPS measurements. C.L. and Z.T. conducted the Raman measurements. C.M. performed the 3D tomography. Z.T. and K.S.N. gave the valuable discussions and suggestions. J.D., D.D. and X.B. co-wrote the manuscript.

\section{Additional information}

Supplementary Information accompanies this paper at http://www.nature.com/ naturecommunications

Competing interests: The authors declare no competing financial interests.

Reprints and permission information is available online at http://npg.nature.com/ reprintsandpermissions/

How to cite this article: Deng, J. et al. Multiscale structural and electronic control of molybdenum disulfide foam for highly efficient hydrogen production. Nat. Commun. 8, 14430 doi: $10.1038 /$ ncomms14430 (2017).

Publisher's note: Springer Nature remains neutral with regard to jurisdictional claims in published maps and institutional affiliations.

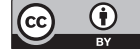

This work is licensed under a Creative Commons Attribution 4.0 International License. The images or other third party material in this article are included in the article's Creative Commons license, unless indicated otherwise in the credit line; if the material is not included under the Creative Commons license, users will need to obtain permission from the license holder to reproduce the material. To view a copy of this license, visit http://creativecommons.org/licenses/by/4.0/

(C) The Author(s) 2017 